\title{
Small collision systems: Theory overview on cold nuclear matter effects
}

\author{
Néstor Armesto ${ }^{1, \star}$ \\ ${ }^{1}$ Departamento de Física de Partículas and IGFAE, Universidade de Santiago de Compostela, \\ 15782 Santiago de Compostela, Galicia-Spain
}

\begin{abstract}
Many observables measured at the Relativistic Heavy Ion Collider and the Large Hadron Collider show a smooth transition between proton-proton and protonnucleus collisions (small systems), and nucleus-nucleus collisions (large systems), when represented versus some variable like the multiplicity in the event. In this contribution I review some of the physics mechanisms, named cold nuclear matter effects, that may lead to a collective-like behaviour in small systems beyond the macroscopic description provided by relativistic hydrodynamics. I focus on the nuclear modification of parton densities, single inclusive particle production and correlations.
\end{abstract}

\section{Motivation}

In recent years much attention has been devoted in the high-energy physics community to the experimental findings that indicate a smooth transition between "small" collisions systems (proton-proton, pp, and proton-nucleus, pA) and "large" collision systems (nucleus-nucleus, AA). These findings at both the Large Hadron Collider (LHC) and the Relativistic Heavy Ion Collider (RHIC), are particularly striking as some of the involved observables have been traditionally considered as signatures of the creation of deconfined partonic matter close to thermodynamical equilibrium - Quark Gluon Plasma - in high-energy AA collisions. They include (see the review [1]) azimuthal asymmetries of apparent collective nature, thermal-like spectra and baryon/strangeness enhancement at small and intermediate transverse momentum respectively, hadrochemistry, interferometry, quarkonium suppression,..., the only exception being jet quenching.

While this behaviour was first observed in AA and high-multiplicity pA and pp collisions, the interest in this subject has been increased recently by the observation that azimuthal asymmetries and the ridge structure - two particle correlations whose strength extends through several units of pseudorapidity and is maximum at zero (near side region) and $\pi$ (back-to-back region) azimuthal angle - can also be observed in pp collisions with multiplicities close to minimum bias [2-4]. From the theoretical side, two facts augment the importance of these findings even further: the fact that viscous relativistic hydrodynamics is able to provide a description of such data [5] even if the conditions of large opacity and approximate momentum isotropy seem difficult to fulfil in pp; and the applicability of hydrodynamics in far-from-equilibrium situations that was obtained both at strong $[6,7]$ and weak coupling [8]. Thus, the traditional statement that the success of hydrodynamics clearly indicated the

\footnotetext{
^e-mail: nestor.armesto@usc.es
} 
existence of an equilibrated partonic medium with vanishing viscosity has moved to the questions [9]:

- What does this apparent continuity in the applicability of collective ideas from small to large systems tell us about the dynamics of a non-equilibrium partonic system in Quantum Chromodynamics (QCD)?;

- How does the macroscopic description that hydrodynamics provides emerge from the microscopic QCD dynamics?

Several reviews exist on the subject, see e.g. $[10,11]$ and also the numerous presentations at the recent Initial Stages conference [12]. The experimental aspects have been presented in the talks by R. Preghenella and N. Novitzky at this conference. The aim of this contribution is to review effects that may cause or contribute to the collectivity found in small systems, whose origin lies in the initial state or the very initial stages of the collision. Therefore, they may provide an alternative or complementary explanation to that of final state rescattering leading to hydrodynamics. And, in any case, such initial state effects, called cold nuclear matter effects (CNM), must be anyway addressed both from a fundamental point of view - long range rapidity correlations in the ridge must come from the early stages of the collision, and from an instrumental one - hydrodynamics must be provided with initial conditions and the effects on the final observables of the dynamics prior to the hydrodynamic behaviour constrained. Due to limitations of space, I will focus on a few aspects: nuclear modification of parton densities, single inclusive particle production and correlations in small systems. References will not be exhaustive. I apologise in advance to those who may find their work underrepresented.

\section{Nuclear modification of parton densities}

The fact that structure functions in bound nucleons are different from those in free nucleons has been known for decades [13]. In collinear factorisation that is the standard tool for the calculation of observables with a perturbative scale in hadronic collisions, this fact reflects in nuclear modifications of parton densities (nPDFs), usually studied through the nuclear modification factor for parton density $f_{i}, R_{i}\left(x, Q^{2}\right)=f_{i}^{A}\left(x, Q^{2}\right) /\left[A f_{i}^{p}\left(x, Q^{2}\right)\right]$. The most recent analysis are shown in Fig. 1.

Focusing on the two most recent ones [14, 15], the difference between them are mainly the degree of freedom for the nuclear effects on the different quark flavours, applied only to valence in [14] and to both valence and sea quarks in [15], and the inclusion of neutrino and pPb LHC data in the second. The difference can be seen in the left and middle plots in Fig. 2. On the other hand, the effect of neutrino and $\mathrm{pPb}$ LHC data can be seen in the plots of the right, through a comparison of [15] with analysis that do not include LHC data [16] or neither neutrino nor LHC data [17]. While parton densities in the different analysis coincide in the regions constrained by experimental data, some comments are in order:

- The effect of the form of the initial parametrisation for DGLAP evolution, widely different in $[14,15]$, reflects in the fact that the uncertainties obtained by the standard Hessian analysis are very different, and also in the behaviours in regions far from those constrained by experimental data e.g. $x<0.01$;

- The effect of including more sets of data, given by the comparison of the results of [15] with those of previous analysis $[16,17]$, seems small and even counterintuitive. This is partly due to the small constraining power of new data except in specific regions (e.g. for the glue at $x \sim 0.1$ ) and by the fact that a new, more flexible initial parametrisation produces larger uncertainties even if the same sets of data are employed.

Both items emphasise the role of the initial parametrisations on the uncertainties and extrapolations of nPDFs, an aspect well known in the community of proton parton densities and that will play an important role in the analyses of nPDFs in the future. 


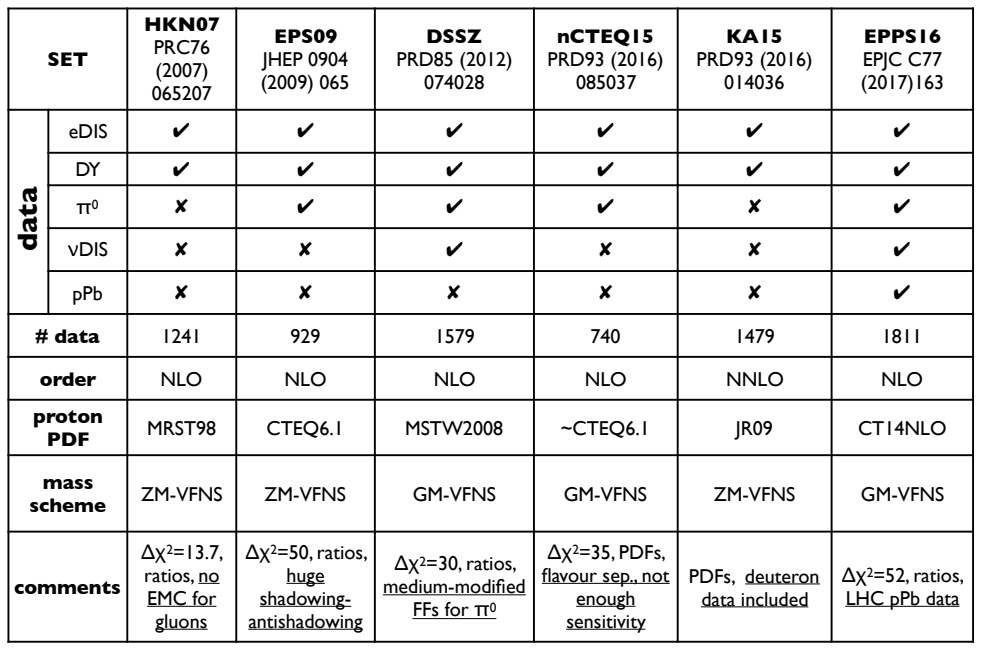

Figure 1. Summary of the most recent global analyses of nPDFs indicating the acronym and reference, the sets of employed data, the order of the perturbative evolution, the reference proton parton density used in the analysis, the scheme for treating heavy flavours and some comments like the employed tolerance and peculiarities of the analysis. See the relevant reference for details.
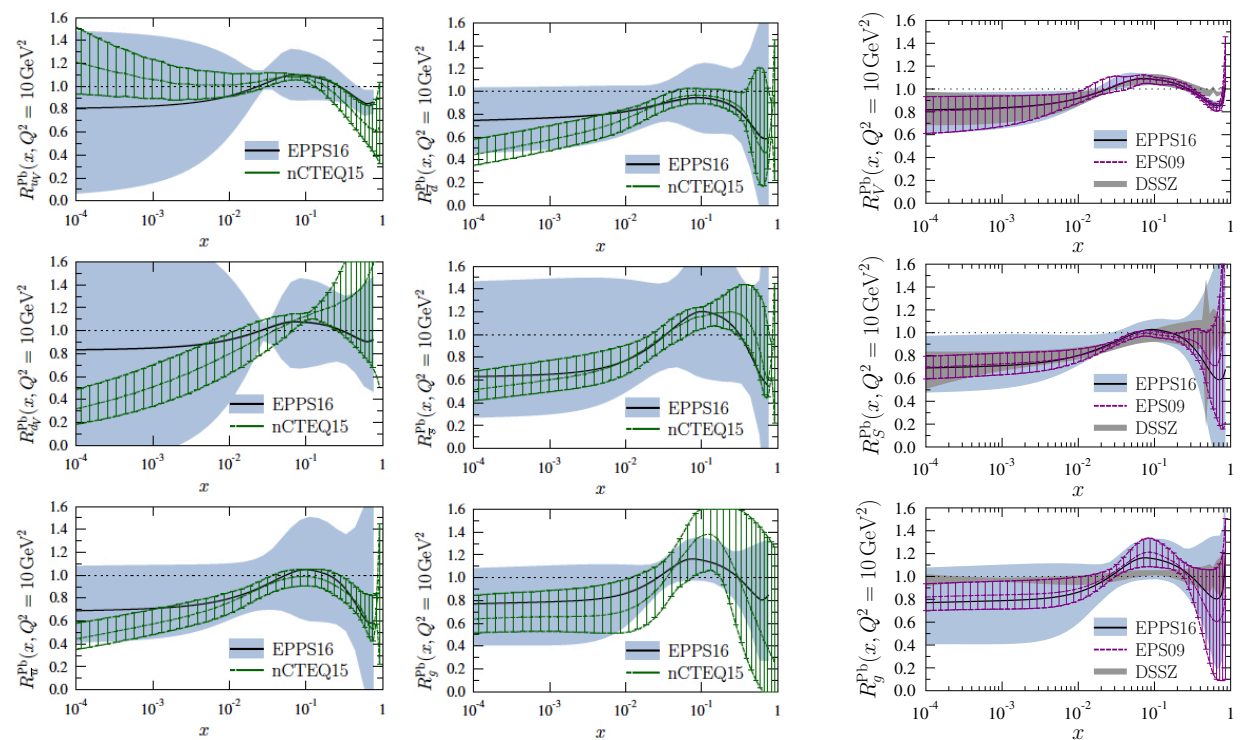

Figure 2. Left and middle plots: comparison between the nuclear modification factors nPDFs from the nCTEQ15 and EPPS16 sets. Plots on the right: id. from the EPPS16, EPS09 and DSSZ sets. 


\begin{tabular}{|c|c|c|c|c|}
\hline Item & Order & Theory & $\begin{array}{l}\text { Pheno- } \\
\text { menology }\end{array}$ & Comments \\
\hline Evolution eqns. & NLO & $\boldsymbol{v}$ & $\sim$ & $\begin{array}{l}\text { rcBK and resummations; dilute-dense } \\
\text { approx. }\end{array}$ \\
\hline DIS impact factor & NLO & $\checkmark$ & $\boldsymbol{X}$ at NLO & dilute-dense approx. \\
\hline Hadrons at $y \sim 0$ & LO & $\checkmark$ & $\checkmark$ & $\mathrm{q}$ and $\mathrm{Q}$, dilute-dense approx. \\
\hline Forward hadrons & NLO & $\checkmark$ & $\checkmark$ & $\mathrm{q}$ and $\mathrm{Q}$, hybrid formalism \\
\hline Quarkonium at $\mathbf{y} \sim 0$ & LO & $\boldsymbol{v}$ & $\boldsymbol{v}$ & dilute-dense approx.+NRQCD \\
\hline Forward quarkonium & LO & $\checkmark$ & $\checkmark$ & hybrid formalism \\
\hline$\gamma^{(*)}$ at $y \sim 0$ & NLO & $\checkmark$ & $\boldsymbol{x}$ at NLO & dilute-dense approx., not yet DY at NLO \\
\hline Forward $\gamma^{(*)}$ & LO & $\checkmark$ & $\checkmark$ & hybrid formalism \\
\hline Dijets at y 0 & LO & $\boldsymbol{v}$ & $\boldsymbol{v}$ & dilute-dense approx., partial NLO \\
\hline Forward dijets & LO & $\checkmark$ & $\boldsymbol{v}$ & $\begin{array}{l}\text { hybrid formalism and high-energy } \\
\text { factorisation, partial NLO }\end{array}$ \\
\hline Diffractive dijets & NLO & $\boldsymbol{v}$ & $\boldsymbol{X}$ at NLO & dilute-dense approx. \\
\hline$g / q / \gamma-g / q / \gamma$ correlations & LO & $\checkmark$ & $v / x$ & $\begin{array}{l}\text { glasma graph approx. + some density } \\
\text { corrections; hybrid formalism }\end{array}$ \\
\hline
\end{tabular}

Figure 3. Summary of processes studied in the CGC with the perturbative order of the calculation, their availability for theory and phenomenology, and some comments.

RHIC and the LHC have also provided data with constraining power on nPDFs through nuclear ultraperipheral collisions (UPCs), see the recent review [18] and refs. therein. While data have clearly established the existence of nuclear gluon shadowing in $\mathrm{Pb}$ [19], uncertainties in the theory of quarkonium production makes it questionable to introduce such data in a global analysis of nPDFs. On the other hand, UPCs offer a huge potential for constraining the transverse partonic structure of the proton, see [20,21].

As a final comment, it should be noted that azimuthal asymmetries and other signals of collectivity cannot be presently described within collinear factorisation. Therefore, the use of low transverse momentum observables from $\mathrm{dAu}$ or $\mathrm{pPb}$ collisions to constrain nPDFs should be carefully examined.

\section{Single inclusive particle production}

Recently, a huge progress has been developed on calculations in the Color Glass Condensate (CGC) [22], the weak coupling but non perturbative effective theory of dense partonic systems. In Fig. 3 I summarise the status of recent calculations. One point that should be stressed is that most calculations have been done in a dilute-dense regime suitable for the study of pp or pA collisions in the forward (proton) rapidity region.

Concerning single inclusive particle production, see Fig. 4, much progress has been made lately to understand and control the next-to-leading corrections (NLO) computed some years ago [24, 25] in the hybrid formalism ${ }^{1}$. This is a complex calculation where collinear and soft divergencies appear. The collinear ones are absorbed in the DGLAP evolution of standard collinear parton densities and fragmentation functions, while the soft ones produce the small- $x$ JIMWLK evolution of the target colour ensemble, both evolutions appearing at leading order (LO). After realising that the NLO results

\footnotetext{
${ }^{1}$ The hybrid formalism considers the scattering of a dilute projectile, whose partons can be treated as collinear, on a dense target that is considered an ensemble of colour fields in the CGC framework.
} 


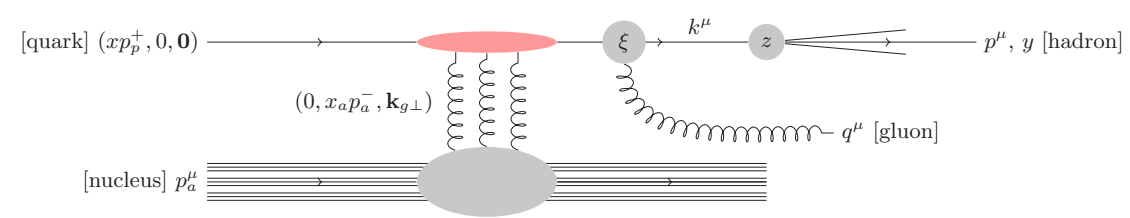

Figure 4. Diagram showing the next-to-leading contribution to single hadron production at forward rapidities in the CGC. Taken from [23].
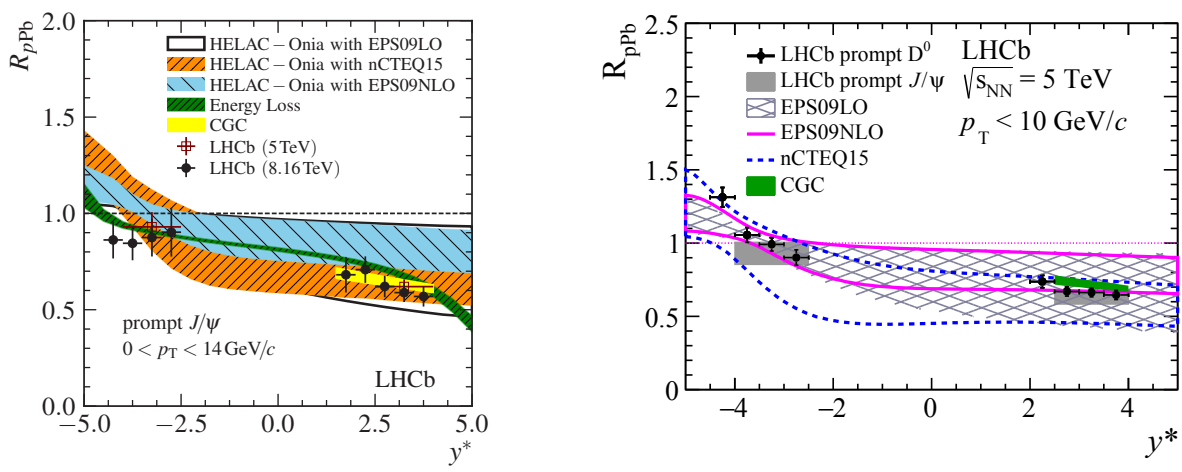

Figure 5. Nuclear modification factor for prompt J/4's (left plot, from [33]) and D's (right plot, from [34]) compared to several models. Those labeled 'CGC' are predictions from the hybrid formalism, those labeled 'Energy Loss' contain final state effects for $\mathrm{J} / \psi$, and the rest are purely collinear approaches using different sets of nPDFs. See the experimental papers for details and references.

turned the spectrum negative at moderate transverse momentum (how large this transverse momentum is depends strongly on the employed parametrisation for the dipole-nucleus scattering amplitude), several solution were proposed. Those fluctuations of the projectile too short lived to be resolved by the target had to be removed [26], which gave a natural way to regularise the soft divergencies and produced additional terms in the production cross section that shifted the negativity problem to larger values of transverse momenta.

While a complete understanding is still under progress, at present the key idea lies is devising a procedure for subtraction of the soft divergencies that is able to maintain the positivity of the cross section while resumming leading higher order terms, see [27] and refs. therein. The problem is related to the large negative corrections found in the NLO JIMWLK evolution equations [28, 29]. Several methods have been proposed for this purpose [30,31]. Meanwhile, the comparison of measurements of the nuclear modification factors in $\mathrm{pPb}$ collisions at the LHC for charged particles at midrapidity [32], and of J/ $\psi$ 's [33] and D's [34] at forward rapidities, are not conclusive as both CGC predictions and collinear calculations (within their large uncertainties) are compatible with existing data.

As in the previous Section, a word of caution is needed in such comparisons, as there may be other effects at play that are included neither in collinear approaches nor in the CGC calculations. Besides the collective effects mentioned there, there may be a contribution from multiple partonic interactions (MPIs), see below, and our understanding of D meson fragmentation and of $\mathrm{J} / \psi$ production still contains sizeable uncertainties. 


\section{Correlations in small systems}

The ridge phenomenon lies at the heart of the present discussions in small systems. In this Section I will review its explanation in the CGC and attempts to model it in the MPI framework, see Section 1.

\subsection{Color Glass Condensate}

All explanations to the ridge phenomenon in the CGC assume that the final state carries the imprint of the correlations in the initial state, and that the CGC wave function is invariant over long rapidity intervals $\Delta y \sim 1 / \alpha_{s}$. Three type of explanations have been proposed:

- "Glasma graphs" [35] that have given rise to a successful phenomenology [36, 37];

- Local anisotropy of target fields [38];

- Spatial variations of partonic density [39].

Concerning the first item, it has been shown that the correlations are due to the Bose enhancement of gluons in the CGC wave function [40], which also give rise to Hanbury-Brown-Twiss correlations $[41,42]$. Therefore, they contain information both about the 'source' size (of order of the inverse of the saturation scale of the target) and about the distributions of 'sources', of order of the target radius. Correspondingly, quarks experience Pauli blocking [43]. Its limitations are the fact that the rescattering with the target, modelled through eikonal Wilson lines, is taken at lowest order, and that the colour correlations in the target are modelled as Gaussian and isotropic, which leads to the fact that two particle correlations are subleading in the number of colours $N_{c}$, to the absence of odd Fourier harmonics and to the fact that the cumulant $c_{2}\{4\}>0$. Work to include high density corrections and overcome these limitations can be found in [44-46], with the appearance of odd harmonics and correlations no longer subleading in $N_{c}$. Concerning the second item, models considering non isotropic colour correlations in the target and domains with oriented chromoelectric fields have been developed, see [47] and refs. therein, with the result that $c_{2}\{4\}$ becomes leading in $N_{c}$ and negative depending on the size of the anisotropy and on the number of domains.

\subsection{Multiple parton interactions}

MPIs - the fact that multiple parton-parton and nucleon-nucleon collisions may be at play in pp, pA and AA collisions at high energies - underlie current models for pp interactions, see [48] and refs. therein. They can be traced back to dual unitarisation models, see [49] for a review. Most Monte Carlo simulators for AA collisions [50,51] contain them in one form or another.

Besides multiple scattering itself, the colour rearragement (CR) between the partons taking part in different parton-parton collisions and the interaction between strings produced in the collision, has proved crucial for reproducing collectivity-like features in small systems. First, CR have been found to describe the baryon/strangeness enhancement at intermediate transverse momentum [52]. Second, string interactions predicted the collectivity features observed in pp collisions at the LHC [53]. Recently, sophisticated models containing both an enhancement of the string tension due to the overlap of the string colour fields, and repulsion among the strings inspired by lattice results, are able to describe the ridge azimuthal distributions [54], see Fig. 6 left. Besides, final state mechanisms (parton rescattering) also produce the azimuthal ridge structure provided a spatially anisotropic system (two separated strings) is considered for initialising the parton transport [55], see Fig. 6 right.

Summarising, small systems have evolved from benchmark to signatures. The experimental finding that they show many of the features found in AA and interpreted as signatures of QGP plus the success of hydrodynamics to describe them, implies that in order to fully interpret the results in AA, we must understand how collectivity is built with increasing system size. Besides nPDFs, I have 

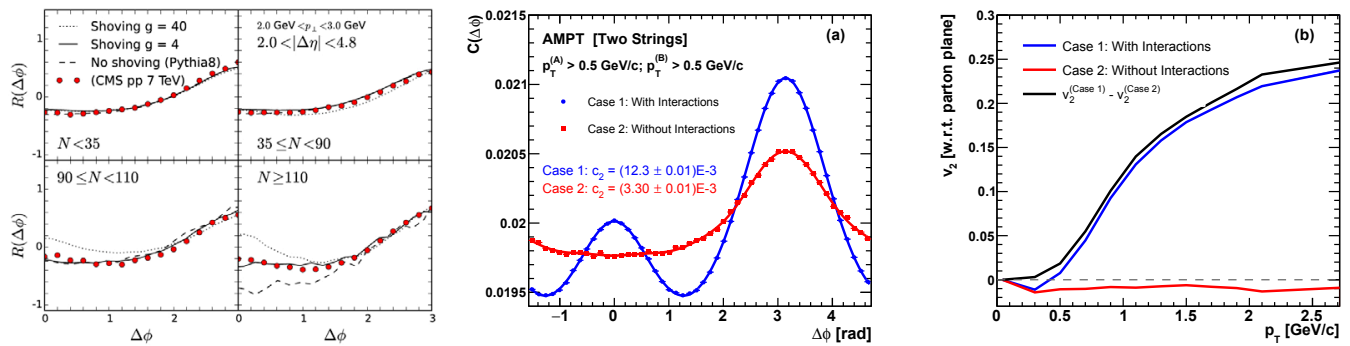

Figure 6. Left plots: two particle azimuthal distributions from the DIPSY model [54] with and without shoving (string repulsion), compared to CMS data. Right plots: two particle azimuthal distributions and second Fourier coefficient in the AMPT model initialised with two strings, with and without parton rescattering [55].

shown some of the CNM alternatives to a hydrodynamical description: the CGC approach to particle production and MPIs. Both contain approximations (e.g. dilute-dense in the CGC) and require nonperturbative input: multiPDFs, models for the colour ensembles in the medium,... The importance of this problem cannot be overstressed as we are addressing a central issue both in QCD and in many other fields: the onset of the transition from the microscopic dynamics to a macroscopic description.

Acknowledgements: I thank the organisers for their invitation and Tolga Altinoluk for a reading of the manuscript. I acknowledge financial support by the ERC grant HotLHC ERC-2011-StG-279579, MiCinn of Spain under project FPA2014-58293-C2-1-P and Unidad de Excelencia María de Maetzu under project MDM2016-0692, Xunta de Galicia (Consellería de Educación) within the Strategic Unit AGRUP2015/11, and FEDER. This work has been performed in the framework of the COST Action CA15213 THOR.

\section{References}

[1] C. Loizides, Nucl. Phys. A 956 (2016) 200.

[2] M. Aaboud et al. [ATLAS Collaboration], Phys. Rev. C 96 (2017) no.2, 024908.

[3] V. Khachatryan et al. [CMS Collaboration], Phys. Lett. B 765 (2017) 193.

[4] M. Aaboud et al. [ATLAS Collaboration], Eur. Phys. J. C 77 (2017) no.6, 428.

[5] R. D. Weller and P. Romatschke, Phys. Lett. B 774 (2017) 351.

[6] P. M. Chesler and L. G. Yaffe, Phys. Rev. D 82 (2010) 026006.

[7] M. P. Heller, R. A. Janik and P. Witaszczyk, Phys. Rev. Lett. 108 (2012) 201602.

[8] A. Kurkela and Y. Zhu, Phys. Rev. Lett. 115 (2015) no.18, 182301.

[9] P. Romatschke, Eur. Phys. J. C 77 (2017) no.1, 21.

[10] K. Dusling, W. Li and B. Schenke, Int. J. Mod. Phys. E 25 (2016) no.01, 1630002.

[11] S. Schlichting and P. Tribedy, Adv. High Energy Phys. 2016 (2016) 8460349.

[12] 4th International Conference on the Initial Stages in High-Energy Nuclear Collisions, Crakow, Poland, September 18th-22nd 2017, https://indico.cern.ch/event/578078/.

[13] M. Arneodo, Phys. Rept. 240 (1994) 301.

[14] K. Kovarik et al., Phys. Rev. D 93 (2016) no.8, 085037.

[15] K. J. Eskola, P. Paakkinen, H. Paukkunen and C. A. Salgado, Eur. Phys. J. C 77 (2017) no.3, 163.

[16] D. de Florian, R. Sassot, P. Zurita and M. Stratmann, Phys. Rev. D 85 (2012) 074028.

[17] K. J. Eskola, H. Paukkunen and C. A. Salgado, JHEP 0904 (2009) 065. 
[18] S. Klein and J. Nystrand, Phys. Today 70 (2017) no.10, 40.

[19] V. Guzey and M. Zhalov, JHEP 1310 (2013) 207.

[20] H. Mäntysaari and B. Schenke, Phys. Lett. B 772 (2017) 832.

[21] J. Cepila, J. G. Contreras and J. D. Tapia Takaki, Phys. Lett. B 766 (2017) 186.

[22] F. Gelis, E. Iancu, J. Jalilian-Marian and R. Venugopalan, Ann. Rev. Nucl. Part. Sci. 60 (2010) 463.

[23] A. M. Staśto, B. W. Xiao, F. Yuan and D. Zaslavsky, Phys. Rev. D 90 (2014) no.1, 014047.

[24] T. Altinoluk and A. Kovner, Phys. Rev. D 83 (2011) 105004.

[25] G. A. Chirilli, B. W. Xiao and F. Yuan, Phys. Rev. D 86 (2012) 054005.

[26] T. Altinoluk, N. Armesto, G. Beuf, A. Kovner and M. Lublinsky, Phys. Rev. D 91 (2015) no.9, 094016.

[27] B. Ducloué, T. Lappi and Y. Zhu, Phys. Rev. D 95 (2017) no.11, 114007.

[28] I. Balitsky and G. A. Chirilli, Phys. Rev. D 77 (2008) 014019.

[29] A. Kovner, M. Lublinsky and Y. Mulian, Phys. Rev. D 89 (2014) no.6, 061704.

[30] E. Iancu, J. D. Madrigal, A. H. Mueller, G. Soyez and D. N. Triantafyllopoulos, Phys. Lett. B 744 (2015) 293.

[31] G. Beuf, Phys. Rev. D 85 (2012) 034039.

[32] B. B. Abelev et al. [ALICE Collaboration], Eur. Phys. J. C 74 (2014) no.9, 3054.

[33] R. Aaij et al. [LHCb Collaboration], Phys. Lett. B 774 (2017) 159.

[34] R. Aaij et al. [LHCb Collaboration], JHEP 1710 (2017) 090.

[35] A. Dumitru, F. Gelis, L. McLerran and R. Venugopalan, Nucl. Phys. A 810 (2008) 91.

[36] K. Dusling and R. Venugopalan, Phys. Rev. D 87 (2013) no.9, 094034.

[37] K. Dusling, P. Tribedy and R. Venugopalan, Phys. Rev. D 93 (2016) no.1, 014034.

[38] A. Kovner and M. Lublinsky, Int. J. Mod. Phys. E 22 (2013) 133000.

[39] E. Levin and A. H. Rezaeian, Phys. Rev. D 84 (2011) 034031.

[40] T. Altinoluk, N. Armesto, G. Beuf, A. Kovner and M. Lublinsky, Phys. Lett. B 751 (2015) 448.

[41] Y. V. Kovchegov and D. E. Wertepny, Nucl. Phys. A 906 (2013) 50

[42] T. Altinoluk, N. Armesto, G. Beuf, A. Kovner and M. Lublinsky, Phys. Lett. B 752 (2016) 113.

[43] T. Altinoluk, N. Armesto, G. Beuf, A. Kovner and M. Lublinsky, Phys. Rev. D 95 (2017) no.3, 034025.

[44] T. Lappi, B. Schenke, S. Schlichting and R. Venugopalan, JHEP 1601 (2016) 061.

[45] A. Kovner, M. Lublinsky and V. Skokov, Phys. Rev. D 96 (2017) no.1, 016010.

[46] K. Dusling, M. Mace and R. Venugopalan, arXiv:1706.06260 [hep-ph].

[47] A. Dumitru, A. V. Giannini and V. Skokov, arXiv:1503.03897 [hep-ph].

[48] T. Sjöstrand, arXiv:1706.02166 [hep-ph].

[49] A. Capella, U. Sukhatme, C. I. Tan and J. Tran Thanh Van, Phys. Rept. 236 (1994) 225.

[50] X. N. Wang and M. Gyulassy, Phys. Rev. D 44 (1991) 3501.

[51] H. J. Drescher, M. Hladik, S. Ostapchenko, T. Pierog and K. Werner, Phys. Rept. 350 (2001) 93.

[52] A. Ortiz Velasquez, P. Christiansen, E. Cuautle Flores, I. Maldonado Cervantes and G. Paić, Phys. Rev. Lett. 111 (2013) no.4, 042001.

[53] L. Cunqueiro, J. Dias de Deus and C. Pajares, Eur. Phys. J. C 65 (2010) 423.

[54] C. Bierlich, G. Gustafson and L. Lönnblad, arXiv:1710.09725 [hep-ph].

[55] J. L. Nagle, R. Belmont, K. Hill, J. Orjuela Koop, D. V. Perepelitsa, P. Yin, Z. W. Lin and D. McGlinchey, arXiv:1707.02307 [nucl-th]. 